\title{
Differentiation between athlete's heart and dilated cardiomyopathy in athletic individuals
}

\author{
Lynne Martina Millar (D , 'Zephryn Fanton, ${ }^{1}$ Gherardo Finocchiaro, ${ }^{1}$ \\ Gabriel Sanchez-Fernandez, ${ }_{1}$ Harshil Dhutia, ${ }^{1}$ Aneil Malhotra, ${ }^{1,2}$ Ahmed Merghani, ${ }^{1}$ \\ Michael Papadakis, ${ }^{1}$ Elijah R Behr, ${ }^{1}$ Nick Bunce, ${ }^{1}$ David Oxborough, ${ }^{3}$ Matthew Reed, ${ }^{1}$ \\ Jamie O'Driscoll, ${ }^{4}$ Maria Teresa Tome Esteban (D) ,' Andrew D'Silva, ${ }^{1}$ \\ Gerry Carr-White, ${ }^{5}$ Jessica Webb, ${ }^{5,6}$ Rajan Sharma, ${ }^{1}$ Sanjay Sharma (D) ${ }^{1}$
}

- Additional material is published online only. To view please visit the journal online (http://dx.doi.org/10.1136/ heartjnl-2019-316147).

For numbered affiliations see end of article.

\section{Correspondence to} Professor Sanjay Sharma, Molecular and Clinical Sciences Research Institute, University of London St George's Molecular and Clinical Sciences Research Institute, London SW17 ORE, UK; sasharma@sgul.ac.uk

Received 21 October 2019 Revised 22 March 2020 Accepted 25 March 2020 Published Online First 27 April 2020

\section{Linked}

- http://dx.doi.org/10.1136/ heartjnl-2020-316979

Check for updates

(c) Author(s) (or their employer(s)) 2020. No commercial re-use. See rights and permissions. Published by BMJ.

To cite: Millar LM, Fanton Z, Finocchiaro $\mathrm{G}$, et al. Heart 2020;106:1059-1065.

\section{ABSTRACT}

Objective Distinguishing early dilated cardiomyopathy (DCM) from physiological left ventricular (LV) dilatation with LV ejection fraction $<55 \%$ in athletes (grey zone) is challenging. We evaluated the role of a cascade of investigations to differentiate these two entities.

Methods Thirty-five asymptomatic active males with DCM, 25 male athletes in the 'grey zone' and 24 male athletes with normal LV ejection fraction underwent $\mathrm{N}$-terminal pro-brain natriuretic peptide (NT-proBNP) measurement, ECG and exercise echocardiography. Grey-zone athletes and patients with DCM underwent cardiovascular magnetic resonance (CMR) and Holter monitoring.

Results Larger LV cavity dimensions and lower LV ejection fraction were the only differences between grey-zone and control athletes. None of the grey-zone athletes had abnormal NT-proBNP, increased ectopic burden/complex arrhythmias or pathological late gadolinium enhancement on CMR. These features were also absent in $71 \%, 71 \%$ and $50 \%$ of patients with DCM, respectively. $95 \%$ of grey-zone athletes and $60 \%$ of patients with DCM had normal ECG. During exercise echocardiography, $96 \%$ grey-zone athletes increased LV ejection fraction by $>11 \%$ from baseline to peak exercise compared with $23 \%$ of patients with DCM $(p<0.0001)$. Peak LV ejection fraction was $>63 \%$ in $92 \%$ grey-zone athletes compared with $17 \%$ patients with DCM $(p<0.0001)$. Failure to increase LV ejection fraction $>11 \%$ from baseline to peak exercise or achieve a peak LV ejection fraction $>63 \%$ had sensitivity of $77 \%$ and $83 \%$, respectively, and specificity of $96 \%$ and $92 \%$, respectively, for predicting DCM.

Conclusion Comprehensive assessment using a cascade of routine investigations revealed that exercise stress echocardiography has the greatest discriminatory value in differentiating between grey-zone athletes and asymptomatic patients with DCM. Our findings require validation in larger studies.

\section{INTRODUCTION}

Dilated cardiomyopathy (DCM) is a rare but recognised cause of sudden cardiac death in athletes. ${ }^{1} \mathrm{~A}$ small proportion of endurance athletes show enlarged left ventricular (LV) cavities with borderline/low left ventricular ejection fraction $(\mathrm{LVEF})^{2}$ which overlaps with the phenotypic expression of morphologically mild DCM. Differentiation between these entities is an important focus of the sports cardiology and imaging societies. Expert opinion suggests that comprehensive assessments including the ECG, advanced imaging such as exercise stress echocardiography, twodimensional strain imaging and the presence of late enhancement on cardiovascular magnetic resonance (CMR) imaging are necessary to differentiate these two entities ${ }^{3}$; however, data regarding utility of such investigations in this context is limited. We sought to evaluate the role of conventional investigations to differentiate between physiological adaptation in healthy athletes with LV dilatation and LVEF $<55 \%$ (grey zone) and active asymptomatic individuals with DCM.

\section{METHODS}

The data, analytical methods and study materials will not be made available to other researchers for the purpose of reproducing the results or replicating the procedure. Researchers interested in the data, methods or analysis can contact the corresponding author for more information. Patients and public were not involved in the design, conduct, reporting or dissemination plans of our research.

\section{Study subjects}

Patients with DCM

Asymptomatic male patients with non-ischaemic DCM were recruited from two tertiary cardiomyopathy centres in London. DCM was defined as systolic impairment in association with LV enlargement (either LV end-diastolic dimension $>58 \mathrm{~mm}$ or LV end-diastolic volume of $>150 \mathrm{~mL}$, equating to $2 \mathrm{SD}$ above the mean, as per the American Society of Echocardiography). ${ }^{5} \mathrm{LV}$ impairment was defined as LVEF $<55 \%$. Exclusion criteria included ischaemic heart disease, hypertension, valvular disease, LVEF $<35 \%$ and poor echocardiographic windows. In individuals who exercised more than 5 hours of exercise per week, DCM was confirmed by the presence of DCM in a first-degree relative, remodelled severe LV systolic dysfunction or late enhancement on CMR. Thirty-five individuals who fulfilled these criteria agreed to participate in the study. 
Healthy athletes with LV dilatation and LVEF <55\% (grey zone)

In the UK, the charity Cardiac Risk in the Young (CRY) subsidises preparticipation cardiovascular evaluations for elite professional and national sporting organisations. Over the period 2015-2017, 8006 athletes were evaluated by CRY. Additionally, the sports cardiology unit at St George's Hospital is a quaternary referral centre for athletes from centres throughout the country. Twenty-five asymptomatic athletes with phenotypic features resembling DCM were recruited from these sources. The grey zone was defined as an athlete with LV enlargement and borderline ejection fraction $(<55 \%)$ who exercised for $\geq 8$ hours per week. Athletes with a family history of DCM were excluded.

\section{Athlete controls}

A control cohort of 24 healthy asymptomatic male athletes with normal LV geometry matched to athletes with an increased LV cavity and LVEF $<55 \%$ for age and sporting discipline were recruited through the CRY screening programme.

\section{Study protocol}

Participants underwent health questionnaire, N-terminal probrain natriuretic peptide (NT-proBNP), 12-lead ECG, baseline and exercise echocardiogram and cardiopulmonary exercise testing (CPET). Beta-blockade was held for 48 hours prior to exercise testing. Grey-zone athletes and patients with DCM also underwent a CMR and 24-hour Holter monitor.

\section{Health questionnaire}

The health questionnaire contained questions regarding cardiovascular symptoms, family history and exercise activity.

\section{NT-proBNP}

Blood samples for NT-proBNP were obtained from participants during resting conditions. Analysis was performed within 2 hours of extraction at room temperature using a Cobas 8000 E602 Module Immunochemistry Analyser (Roche Diagnostics, Basel, Switzerland).

\section{Electrocardiography}

12-lead ECG was performed in the supine position in a quiet room using a GE Marquette Hellige (Milwaukee, Wisconsin, USA) ECG machine with a paper speed of $25 \mathrm{~mm} / \mathrm{s}^{\text {as described. }}{ }^{6}$ ECGs were interpreted in accordance with international guidelines. $^{7}$

\section{Twenty-four-hour Holter}

Twenty-four-hour ambulatory ECG monitoring was performed using Life Card CF Holters (Spacelabs Healthcare). A high ventricular ectopic burden $>500$ beats/24 hours ${ }^{8}$ or the presence of non-sustained ventricular tachycardia (NSVT) was considered abnormal. The presence of NSVT was defined as three or more consecutive beats of $>120 \mathrm{~ms}^{9}$

\section{Echocardiography}

Two-dimensional transthoracic echocardiography was performed by two board accredited sonographers using a commercially available, portable ultrasound system (Vivid E9, GE Healthcare, Milwaukee, Wisconsin, USA) with a $1.5-3.6 \mathrm{MHz}$ phased array transducer. Conventional views were obtained and measurements made as per the American Society of Echocardiography. ${ }^{5}$ Pulsed-wave Doppler recordings were obtained to assess transmitral Doppler, and tissue Doppler imaging (TDI) was acquired at the lateral and septal mitral annulus. ${ }^{10} \mathrm{M}$-mode echocardiography was used to assess the tricuspid annular plane systolic excursion.

\section{Speckle tracking imaging}

Speckle tracking imaging was performed using a designated speckle tracking package (GE EchoPAC Clinical Workstation Software (Pollards Wood, UK)) to obtain global LV longitudinal strain (GLS) in the two-, three-, four-chamber views and then averaged accordingly. A normal GLS value was $<-17 \% .^{5}$

\section{Stress echocardiography}

Exercise echocardiography was conducted on a semirecumbent cycle ergometer (Lode Angio with Echo Cardiac Stress Table, Groningen, The Netherlands) according to a ramp protocol of $20 \mathrm{~W} / \mathrm{min}$ to volitional exhaustion. Standard apical, parasternal short and long-axis images and transmitral Doppler and TDI of the lateral wall were acquired at baseline and peak exercise. LV volumes and ejection fraction were calculated using the Simpson's biplane method. ${ }^{5}$ Intravenous contrast was not required as all subjects had good endocardial definition.

\section{Cardiopulmonary exercise testing}

CPET was performed in an upright position with a COSMED E100w cycle ergometer (Rome, Italy) using a ramp protocol $20-30 \mathrm{~W} / \mathrm{min}$ to volitional exhaustion. Breath-by-breath gas exchange analysis was performed using a dedicated COSMED Quark CPEX metabolic cart (Rome, Italy). Peak oxygen consumption $\left(\mathrm{pVO}_{2}\right)$ was calculated in millilitre per kilogram per minute.

\section{Cardiovascular magnetic resonance}

CMR imaging was performed using methods described and analysed using semiautomated software. ${ }^{11}$ All measurements were recorded as absolute values and indexed to body surface area as per the DuBois-DuBois formula. ${ }^{12}$ Delayed enhancement images were acquired after the administration of gadolinium diethylenetriamine pentaacetate. Isolated late gadolinium enhancement (LGE) at the right ventricular insertion was not considered pathological as this is a common finding in healthy endurance athletes. $^{8}$

\section{Statistical analysis}

Analyses were performed using SPSS (V.25.0, IBM). ShapiroWilk test and analysis of histograms were performed to assess for normality. Continuous variables are presented as mean $\pm \mathrm{SD}$ or median and IQRs. Comparison of two groups was performed by unpaired Student's t-tests or Mann-Whitney U tests. Comparisons of more than two groups were performed by one-way analysis of variance (with Bonferroni post hoc test) or Krushal-Wallis (with Dunn's post hoc test) test where appropriate. Categorical variables were presented as percentages and were compared using Fisher's exact tests or $\chi^{2}$ test. Receiver operating characteristic (ROC) curve analysis was performed to test the sensitivity of the echocardiographic variables in predicting DCM. Athlete was considered a negative test, whereas DCM was considered a positive test. Optimal cut-off values, defined by the best compromise between sensitivity and specificity, were calculated by the Youden's index using Medcalc 19.0.7. Inter-reader variability was assessed by intraclass correlation coefficients. Statistical significance was defined for $p$-values $<0.05$. Forward stepwise logistic regression was used. Stress echocardiographic variables 
with an area under the curve (AUC) $>0.7$ as identified by the ROC curve were included in the model.

To determine sample sizes, we estimated using a previous study of exercise radionuclide angiography which showed those with contractile reserve (representing athletes) had an increase in LVEF of $5 \% \pm 6 \%$ and those with poor outcome (representing DCM) had a change of LVEF of $0 \pm 5 \% .{ }^{13}$ Using these assumptions, we calculated that we needed at least 21 in each cohort to provide $80 \%$ power. To allow for a margin of error, we aimed to recruit at least 30 patients with DCM and match them for age and baseline LVEF with the grey-zone athletes $(\alpha=5 \%$, $1-\beta=80 \%, n=21$ ).

\section{RESULTS}

\section{Demographics}

Patients with DCM

Patients with DCM were aged $39.5 \pm 13.4(18-68)$ years. The majority $(88.6 \%)$ were Caucasians. All patients were in New York Heart Association Class 1 and exercised for an average of 4 (2-8) hours per week. Twenty-four (68.8\%) were on betablockers and $23(65.7 \%)$ on ACE inhibitors or angiotensin II receptor blockers. Three patients $(8.6 \%)$ had an implantable cardioverter-defibrillator in situ. Fifteen patients (42.9\%) had familial DCM, 3 (8.6\%) had anthracycline-induced DCM, 4 (11.4\%) had postviral DCM and 15 (42.9\%) had idiopathic DCM.

\section{Athletes}

Athletes with an enlarged LV and baseline LVEF $<55 \%$ (grey-zone athletes) (32.3 \pm 10.4 ; range 18-58 years) and control athletes (36.7 $\pm 7.7 ; 22-48$ years) were of similar age; however, greyzone athletes were younger than patients with DCM $(p=0.035)$. The majority $(>90 \%)$ were Caucasians. Grey-zone athletes and control athletes exercised for a mean of $14.0(10-20)$ and 10 (8.5-14.75) hours per week, respectively, and participated primarily in endurance sports. Grey-zone athletes participated in cycling $(\mathrm{n}=8)$, endurance running $(\mathrm{n}=10)$, triathlon $(\mathrm{n}=3)$, rowing $(n=3)$ and rugby $(n=1)$. Control athletes competed in cycling $(n=15)$, triathlon $(n=2)$, endurance running $(n=6)$ and rowing $(n=1)$.

\section{Electrocardiography}

All participants were in sinus rhythm. Fourteen (40\%) patients with DCM had an abnormal ECG (some with multiple abnormalities) compared with 2 (8.0\%) grey-zone athletes and 1 (4.2\%) control athlete $(p=0.0007)$. Among the DCM cohort, four had left bundle branch block, two had pathological Q waves, two had ST-segment depression, five had T-wave inversion and four had two or more ventricular extrasystoles. None of these abnormalities were seen in either athletic cohort. Ten (28.6\%) patients with DCM had an abnormal Holter of which $5(14.3 \%)$ showed $>500$ ventricular extrasystoles, $2(5.7 \%)$ revealed isolated NSVT and $3(8.5 \%)$ had both. None of the grey-zone athletes had an abnormal Holter.

\section{NT-proBNP}

There was no significant difference in median NT-proBNP between the three groups $(50(26-262) \mathrm{pg} / \mathrm{mL}$ in the DCM group, $33.0(23.5-57.5) \mathrm{pg} / \mathrm{mL}$ in the grey zone and $28(17.5-$ 42) $\mathrm{pg} / \mathrm{mL}$ in the athlete controls $(\mathrm{p}=0.131))$. Ten $(28.6 \%)$ patients with DCM had an NT-proBNP $>125 \mathrm{pg} / \mathrm{mL}$ (upper limit of normal) ${ }^{14}$ compared with none of the athletes.
Table 1 Baseline echocardiographic characteristics

\begin{tabular}{|c|c|c|c|c|}
\hline & DCM $(n=35)$ & $\begin{array}{l}\text { Healthy athletes } \\
\text { with LV dilatation } \\
\text { and LVEF }<55 \% \\
\text { grey zone }(n=25)\end{array}$ & $\begin{array}{l}\text { Athlete } \\
\text { controls } \\
(n=24)\end{array}$ & $P$ value \\
\hline LAVi $\left(\mathrm{mL} / \mathrm{m}^{2}\right)$ & $29.2(24.4-35.2)$ & $33.7(30.0-37.5)$ & $\begin{array}{l}35.6(31.3- \\
40.7)\end{array}$ & 0.081 \\
\hline LVEDD (mm) & $60.3 \pm 2.2^{*}$ & $59.3 \pm 2.3^{*}$ & $53.3 \pm 3.3$ & $<0.0001$ \\
\hline LVEDD/BSA & $28.6 \pm 3.6$ & $29.8 \pm 2.0$ & $28.2 \pm 2.7$ & 0.137 \\
\hline LVESD (mm) & $45.7 \pm 5.5^{*}$ & $41.8 \pm 3.4^{*}$ & $35.3 \pm 3.7$ & $<0.0001$ \\
\hline LVESD/BSA & $21.7 \pm 3.6$ & $21.0 \pm 2.2$ & $18.7 \pm 2.4$ & $<0.0001$ \\
\hline LV mass (g) & $209.8 \pm 58.1$ & $200.3 \pm 47.9$ & $180.6 \pm 30.4$ & 0.081 \\
\hline Baseline LVEDV (mL) & $185.27 \pm 31.2^{*}$ & $185.0 \pm 20.4^{*}$ & $152.4 \pm 22.9$ & $<0.0001$ \\
\hline Baseline LVESV (mL) & $97.9 \pm 22.8^{*}$ & $92.7 \pm 12.0^{*}$ & $64.4 \pm 11.7$ & $<0.0001$ \\
\hline Baseline SV (mL) & $87.3 \pm 16.3$ & $92.6 \pm 12.0$ & $88.1 \pm 13.7$ & 0.346 \\
\hline $\begin{array}{l}\text { LV ejection fraction } \\
(\%)\end{array}$ & $47.6 \pm 5.4^{*}$ & $49.9 \pm 2.5^{*}$ & $58.3 \pm 2.3$ & $<0.0001$ \\
\hline TAPSE (mm) & $22.2 \pm 4.0$ & $23.6 \pm 3.2$ & $24.5 \pm 4.1$ & 0.059 \\
\hline RVD1 (mm) & $40.2 \pm 5.6 \ddagger$ & $45.4 \pm 4.6$ & $41.4 \pm 5.0 \ddagger$ & 0.001 \\
\hline RVD2 (mm) & $27.6 \pm 5.1$ & $31.9 . \pm 5.5$ & $29.5 \pm 5.5$ & 0.010 \\
\hline Mitral E wave $(\mathrm{cm} / \mathrm{s})$ & $0.71 \pm 0.20$ & $0.52 \pm 0.15$ & $0.88 \pm 0.17$ & 0.487 \\
\hline Mitral A wave $(\mathrm{cm} / \mathrm{s})$ & $0.52 \pm 0.15$ & $0.44 \pm 0.14$ & $0.46 \pm 0.10$ & 0.094 \\
\hline Mitral E/A ratio & $1.53 \pm 0.62$ & $1.97 \pm 0.66$ & $1.93 \pm 0.97$ & 0.096 \\
\hline Lateral $\mathrm{E}^{\prime}(\mathrm{cm} / \mathrm{s})$ & $13.7 \pm 4.8$ & $17.2 \pm 4.4 \dagger$ & $17.1 \pm 3.3 \dagger$ & 0.008 \\
\hline Lateral $\mathrm{S}^{\prime}(\mathrm{cm} / \mathrm{s})$ & $8.8 \pm 2.3$ & $11.4 \pm 2.3 \dagger$ & $11.7 \pm 1.9 \dagger$ & $<0.0001$ \\
\hline Lateral E/E' & $5.99 \pm 2.32$ & $4.33 \pm 1.35 t$ & $4.53 \pm 1.03 \dagger$ & 0.004 \\
\hline Average $\mathrm{E} / \mathrm{E}^{\prime}$ & $6.75 \pm 1.91$ & $5.24 \pm 1.61 \dagger$ & $5.30 \pm 1.26 \dagger$ & 0.007 \\
\hline \multicolumn{5}{|c|}{$\begin{array}{l}\text { *Non-significant between the patients with DCM and athletes in the grey zone. } \\
\text { +Non-significant between grey-zone and control athletes. } \\
\text { fNon-significant between DCM and athlete controls. } \\
\text { BSA, body surface area; DCM, dilated cardiomyopathy; LA, left atrial; LAVi, left atrial } \\
\text { volume indexed; LV, left ventricular; LVEDD, left ventricular end-diastolic dimension; LVEDV, } \\
\text { left ventricular end-diastolic volume; LVEF, left ventricular ejection fraction; LVESD, left } \\
\text { ventricular end-systolic dimension; LVESV, left ventricular end-systolic volume; RVD1, right } \\
\text { ventricular basal dimension; RVD2, right ventricular mid-cavity dimension; RVD3, right } \\
\text { ventricular longitudinal dimension 3; SV, stroke volume; TAPSE, tricuspid annular plane } \\
\text { systolic excursion. }\end{array}$} \\
\hline
\end{tabular}

\section{Baseline echocardiography}

There were no significant differences in the LV end-diastolic dimensions or ejection fraction between grey-zone athletes or patients with DCM. Both groups had a larger LV cavity compared with control athletes, but there were no differences between the groups in left atrial indexed volume or LV mass (Table 1). LA dilatation was observed in $12(48.0 \%)$ grey-zone athletes, $16(66.7 \%)$ control athletes and $12(34.3 \%)$ patients with DCM. Diastolic dysfunction was noted in five patients with DCM (two grade I, two grade II and one grade III). None of the athletes had diastolic dysfunction. Both athletic cohorts showed significantly higher TDI measurements compared with patients with DCM. Lateral $S^{\prime}$ wall was higher in both athletic groups compared with patients with DCM. All the grey-zone athletes and $28(80.0 \%)$ patients with DCM had a lateral $\mathrm{E}^{\prime} \geq 10 \mathrm{~cm} / \mathrm{s}$. Twenty (80\%) grey-zone athletes and 15 (42.9\%) patients with DCM had an $S^{\prime}$ wave $\geq 10 \mathrm{~cm} / \mathrm{s}$.

\section{Speckle tracking imaging}

Average GLS was highest in athlete controls $(-17.4 \% \pm 1.9 \%)$, followed by grey-zone athletes $(-16.0 \% \pm 2.1 \%)$ and patients with DCM $(-13.6 \% \pm 3.0 \%), \mathrm{p}<0.0001$. A significant proportion of grey-zone athletes $(\mathrm{n}=17 ; 68 \%), 14(\mathrm{n}=14 ; 58.3 \%)$ control athletes and $27(n=27 ; 79.4 \%)$ patients with DCM had GLS values outside the normal range $(<-17 \%) .^{5}$ 
Table 2 Stress echocardiographic characteristics

\begin{tabular}{|c|c|c|c|c|}
\hline & DCM $(n=35)$ & $\begin{array}{l}\text { Athlete in the } \\
\text { grey zone } \\
(n=25)\end{array}$ & $\begin{array}{l}\text { Athlete } \\
\text { controls }(n=24)\end{array}$ & $P$ value \\
\hline Total watts & $234.6 \pm 48.0$ & $308.6 \pm 59.6^{*}$ & $293.5 \pm 59.6^{*}$ & $<0.0001$ \\
\hline Peak LVEDV (mL) & $176.3 \pm 40.3 \dagger$ & $167.4 \pm 17.5 \dagger$ & $140.7 \pm 22.8$ & $<0.0001$ \\
\hline Peak LVESV (mL) & $86.2 \pm 34.7 \dagger$ & $56.2 \pm 11.3 \dagger$ & $40.3 \pm 4.9$ & $<0.0001$ \\
\hline Peak SV (mL) & $90.1 \pm 22.8$ & $111.2 \pm 15.6$ & $101.8 \pm 17.9$ & $<0.0001$ \\
\hline $\begin{array}{l}\text { Peak LV ejection } \\
\text { fraction (\%) }\end{array}$ & $52.0 . \pm 11.5$ & $67.6 \pm 3.9^{*}$ & $71.4 \pm 3.4^{*}$ & $<0.0001$ \\
\hline $\begin{array}{l}\text { Change in LV } \\
\text { ejection fraction } \\
(\%)\end{array}$ & $4.9 \pm 8.9$ & $17.7 \pm 4.1$ & $13.1 \pm 3.1$ & $<0.0001$ \\
\hline $\begin{array}{l}\text { Peak mitral E } \\
\text { wave }\end{array}$ & $1.34 \pm 0.28$ & $1.30 \pm 0.27$ & $1.46 \pm 0.24$ & 0.217 \\
\hline $\begin{array}{l}\text { Peak Lateral E' } \\
(\mathrm{cm} / \mathrm{s})\end{array}$ & $21.5 \pm 5.5$ & $23.6 \pm 5.2$ & $23.8 \pm 5.8$ & 0.266 \\
\hline Peak Lateral E/E' & $6.6 \pm 2.3$ & $5.9 \pm 2.0$ & $5.86 \pm 1.84$ & 0.463 \\
\hline Peak S' $(\mathrm{cm} / \mathrm{s})$ & $15.6 \pm 5.0$ & $22.1 \pm 6.1^{*}$ & $22.5 \pm 6.6^{*}$ & $<0.0001$ \\
\hline $\begin{array}{l}\text { Peak SBP (mm } \\
\mathrm{Hg})\end{array}$ & $189.5 \pm 26.7$ & $210.3 \pm 24.7^{*}$ & $202.3 \pm 27.2^{*}$ & 0.007 \\
\hline $\begin{array}{l}\text { Peak DBP (mm } \\
\mathrm{Hg})\end{array}$ & $98.0 \pm 11.0$ & $102.3 \pm 13.9$ & $94.1 \pm 14.50$ & 0.018 \\
\hline Peak HR (bpm) & $148.6 \pm 15.4 \ddagger$ & $162.2 \pm 11.1$ & $150.6 \pm 9.7 \ddagger$ & 0.01 \\
\hline
\end{tabular}

*Non-significant between grey zone and control athletes.

tNon-significant between grey zone and DCM.

‡Non-significant between DCM and athlete controls.

bpm, beats per minute; DBP, diastolic blood pressure; DCM, dilated cardiomyopathy; $H R$, heart rate; LV, left ventricular; LVEDV, left ventricular end-diastolic volume; LVESV, left ventricular end-systolic volume; SBP, systolic blood pressure; SV, stroke volume.

\section{Exercise echocardiogram}

All cohorts demonstrated improvement in indices of diastolic $\left(E^{\prime}\right)$ and longitudinal systolic function $\left(S^{\prime}\right)$ at peak exercise; however, the athletes showed a greater improvement in lateral $\mathrm{S}^{\prime}$ compared with patients with DCM (table 2). Change in LVEF $\leq 11 \%$ and peak LVEF $\leq 63 \%$ were considered the optimal 'cut-off' to distinguish between DCM and grey-zone athletes (table 3). All but one of the grey-zone athletes (96.0\%) failed to increase LVEF $>11 \%$ as did $19(79.2 \%)$ control athletes (figure 1) compared with only 8 (22.9\%) patients with DCM. All athlete controls and 23 (92.0\%) grey-zone athletes achieved a peak LVEF $>63 \%$ compared with only $6(17.1 \%)$ patients with DCM (figure 2). Thirty (85.7\%) patients with DCM failed to increase LVEF by $>11 \%$ or achieve a peak ejection fraction $>63 \%$. Combining the inability to achieve a peak exercise LVEF $>63 \%$ and a change in LVEF $>11 \%$ exercise echocardiography had a sensitivity of $85.7 \%$ and specificity of $92.0 \%$.

\section{Cardiovascular magnetic resonance}

All but one patient with DCM and one athlete with a dilated LV and LVEF $>55 \%$ (grey zone) underwent a CMR. Pathological LGE was observed in 17 (50.0\%) patients with DCM (mid wall $n=12$ and subepicardial $n=5$ ) compared with none of the greyzone athletes (online supplementary table 1 ).

\section{Cardiopulmonary exercise testing}

There were no significant differences in cardiopulmonary parameters between either athletic group and both achieved superior results compared with patients with DCM (online supplementary table 2$)$. A significant proportion $(n=25 ; 71.4 \%)$ of patients with DCM had a normal $\mathrm{pVO}_{2}{ }^{15}$ with seven (20\%) achieving a $\mathrm{pVO}_{2}$ of $>120 \%$ predicted. Of these seven, all had ventricular arrhythmias on Holter and six had the late enhancement on CMR.

\section{Discriminating ability of echocardiographic parameters}

ROC curve analysis showed peak LVEF $\leq 63 \%$ (AUC 0.904; $\mathrm{p}<0.0001$ ) and change LVEF $\leq 11 \%$ (AUC 0.906; $\mathrm{p}<0.0001$ ) predicted DCM with good sensitivity and excellent specificity (table 3). Stepwise logistic regression model including a change in $\mathrm{LVEF} \leq 11 \%$, peak LVEF $\leq 63 \%$, peak stroke volume $\leq 94 \mathrm{~mL}$ and peak $\mathrm{S}^{\prime} \leq 21 \mathrm{~cm} / \mathrm{s}$ as predictors of DCM revealed that a change in LVEF $\leq 11 \%$ independently predicted DCM. The final model had a Nagelkerke $\mathrm{R}^{2}$ of 0.677 .

\section{Interobserver variability}

Agreement between observers for the echocardiographic variables was assessed on a random sample of 40 stress echocardiograms using intraclass coefficient between the primary observer and an independent observer blinded to the initial readings and other results. The intraclass coefficients for the assessment of baseline LVEF, the difference between baseline and peak LVEF and peak LVEF were $0.734,0.877$ and 0.899 , respectively.

\section{DISCUSSION}

To our knowledge, this is the first study which has comprehensively assessed the utility of a cascade of investigations to differentiate between the athletes with an enlarged LV and LVEF $<55 \%$ (grey zone) and morphologically mild DCM. Our results reveal the combination of investigations including NT-proBNP, ECG, Holter and CMR that will fail to diagnose DCM $>30 \%$ of cases. Whereas NT-proBNP $>125 \mathrm{pg} / \mathrm{mL}$ was highly specific for DCM, most affected active patients had normal values. The ECG has a sensitivity of $90 \%{ }^{16}$ and $80 \%{ }^{17}$ in hypertrophic and arrhythmogenic cardiomyopathy, respectively; however, only $40 \%$ of our active individuals with DCM demonstrated abnormal ECGs. ${ }^{7}$ Although, beyond the scope of this paper, genetic testing may

Table 3 Receiver operator characteristic curve analysis evaluating biomarkers and structural and functional stress echocardiographic parameters to distinguish between dilated cardiomyopathy and athletic adaptation

\begin{tabular}{|c|c|c|c|c|c|}
\hline Variable & Optimal 'cut-off'* & AUC & Sensitivity & Specificity & $P$ value \\
\hline NT-proBNP & $>75 \mathrm{pg} / \mathrm{mL}$ & 0.645 & $48.6 \%$ & $96.0 \%$ & 0.045 \\
\hline$E^{\prime}$ lateral peak & $<25 \mathrm{~cm} / \mathrm{s}$ & 0.638 & $78.8 \%$ & $48.0 \%$ & 0.066 \\
\hline$S^{\prime}$ lateral peak & $\leq 21 \mathrm{~cm} / \mathrm{s}$ & 0.792 & $84.4 \%$ & $64.0 \%$ & $<0.001$ \\
\hline Stroke volume peak & $\leq 94 \mathrm{~mL}$ & 0.754 & $62.9 \%$ & $96.0 \%$ & $<0.001$ \\
\hline LV ejection fraction & $\leq 63 \%$ & 0.904 & $82.9 \%$ & $92.0 \%$ & $<0.0001$ \\
\hline Change in LV ejection fraction from baseline to peak exercise & $\leq 11 \%$ & 0.906 & $77.1 \%$ & $96.0 \%$ & $<0.0001$ \\
\hline
\end{tabular}

*Value calculated by Youden's index as best compromise between sensitivity and specificity.

AUC, area under the curve; LV, left ventricular; NT-proBNP, N-terminal pro-brain natriuretic peptide. 

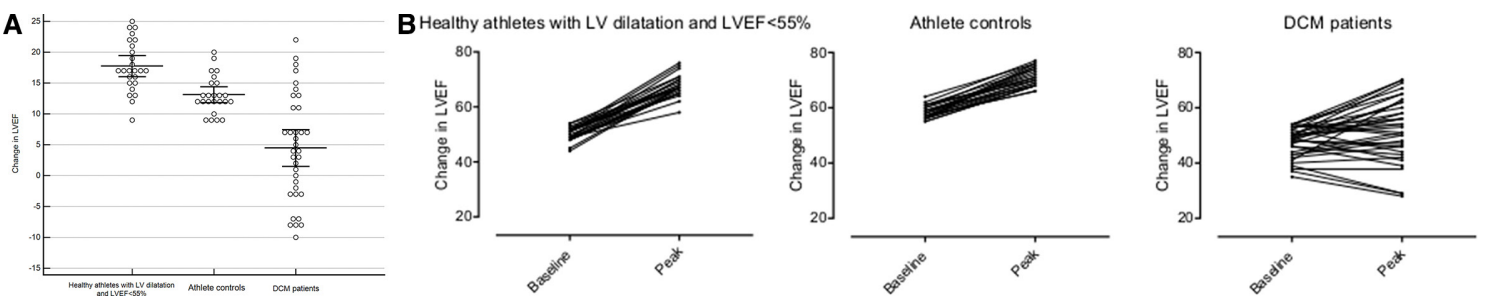

Figure 1 (A) Change in left ventricular ejection fraction (LVEF) from baseline to peak exercise in the healthy athletes with left ventricular (LV) dilatation and LVEF $<55 \%$ (the grey zone) (left), athlete controls (centre) and individuals with morphologically mild dilated cardiomyopathy (DCM) (right). Each circle represents an individual and the horizontal line represents the mean and the $95 \%$ Cls. Almost all the athletes in both cohorts increase the LVEF by $>11 \%$ compared with the DCM cohort who demonstrate a heterogeneous response. (B) The change in ejection fraction from baseline to peak exercise. The healthy athletes with LV dilatation and LVEF $<55 \%$ (grey zone) are on the left, the DCM cohort on the right and the control athletes in the centre. All the athletes demonstrate an increase in LVEF compared with the patients with DCM who show a heterogeneous response.

have a role in resolving this diagnostic conundrum; however, it is limited by the relatively high cost and low yield for results.

\section{Indices of diastolic and longitudinal function}

Baseline echocardiographic markers of systolic and diastolic function as assessed by $\mathrm{E}^{\prime}$ and $\mathrm{S}^{\prime}$ at the lateral mitral annulus had a sensitivity of $51.4 \%$ and $88.6 \%$, respectively, in differentiating between grey-zone athletes and patients with DCM. Although GLS was higher in the grey-zone athletes compared with patients with DCM, over 50\% had low values. ${ }^{5}$ Interpretation of these results is challenging because currently there is no clear consensus on 'normal' GLS values in athletes with a borderline or low LVEF. A meta-analysis by Beaumont et $a l^{18}$ reported that GLS values in athletes ranged from $-16.5 \%$ to $-23.3 \%$ and were lower in endurance athletes. Our results suggest that GLS may be of limited value in this context as low values may not be pathological. Further research is required on the spectrum of GLS values in endurance athletes with borderline or mildly depressed LV function at rest.

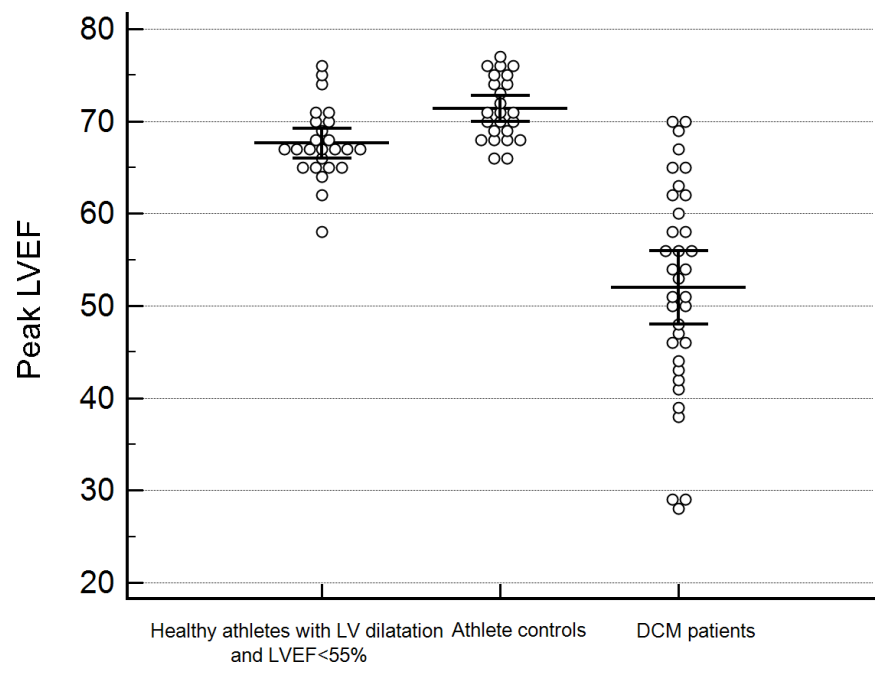

Figure 2 Peak exercise left ventricular ejection fraction (LVEF). This figure shows peak exercise LVEF from baseline to peak exercise in the healthy athletes with left ventricular (LV) dilatation and LVEF $<55 \%$ (grey zone) (left), control athletes (centre) and dilated cardiomyopathy (DCM) cohort (right). Each circle represents an individual and the horizontal line represents the mean and the $95 \% \mathrm{Cl}$. All the athlete controls and almost all the grey-zone athletes increase their LVEF to $>63 \%$ from baseline to peak exercise, which is in contrast to the DCM cohort.

\section{Exercise stress echocardiography}

Our results demonstrate the importance of exercise echocardiography in differentiating between these entities. Failure to increase LVEF by $>11 \%$ from baseline to peak exercise is a useful marker of impaired contractile reserve. Only six patients with DCM were able to generate an LVEF > 63\% at peak exercise compared with more than $90 \%$ of the grey-zone athletes and all the athletic controls and therefore the inability to achieve a peak LVEF $>63 \%$ is an additional marker of pathology. The sensitivity of either of these parameters was around $80 \%$ and the specificity was around 90\%. Combining these parameters to define a 'normal' test reduces the false negatives to $5(14.2 \%)$ with only 2 (8\%) false positive results.

There are limited data used to define contractile reserve in health and this has predominantly focused on pharmacological and non-echocardiographic methods. ${ }^{13} 1920$ We used exercise echocardiography as it is more physiological and exercise echocardiography is readily available to the physician. Our findings are in-keeping recent study using exercise CMR which also found that a failure to increase LVEF by $>11 \%$ at peak exercise predicted DCM. ${ }^{21}$

\section{Cardiopulmonary exercise testing}

Although all but one of the grey-zone athletes showed normal $\mathrm{pVO}_{2}$, we observed normal $\mathrm{pVO}_{2}$ in three-quarters of the DCM cohort. Additionally, superior $\mathrm{pVO}_{2}>120 \%$ predicted was seen in a fifth of our cohort which is similar to a published study looking athletes with hypertrophic cardiomyopathy. ${ }^{22}$ All the individuals with a $\mathrm{pVO}_{2}>120 \%$ predicted had ventricular arrhythmias and most had late enhancement on CMR. Therefore, highly trained individuals may have excellent functional capacity despite significant pathology

\section{Cardiovascular magnetic resonance}

In our study, CMR identified pathological LGE in only 50\% patients with DCM, suggesting that baseline CMR at rest is not enough on its own to exclude pathology which gives further importance to the role of stress echocardiography in this setting. Although we did not use T1 and T2 mapping techniques, data suggest these techniques may be useful in distinguishing athlete's heart from DCM. ${ }^{23}$

\section{Algorithm}

Based on our findings, we have produced a clinical algorithm with diagnostic thresholds to aid physicians when assessing highly active individuals with a dilated LV and an LVEF $<55 \%$ (figure 3) 


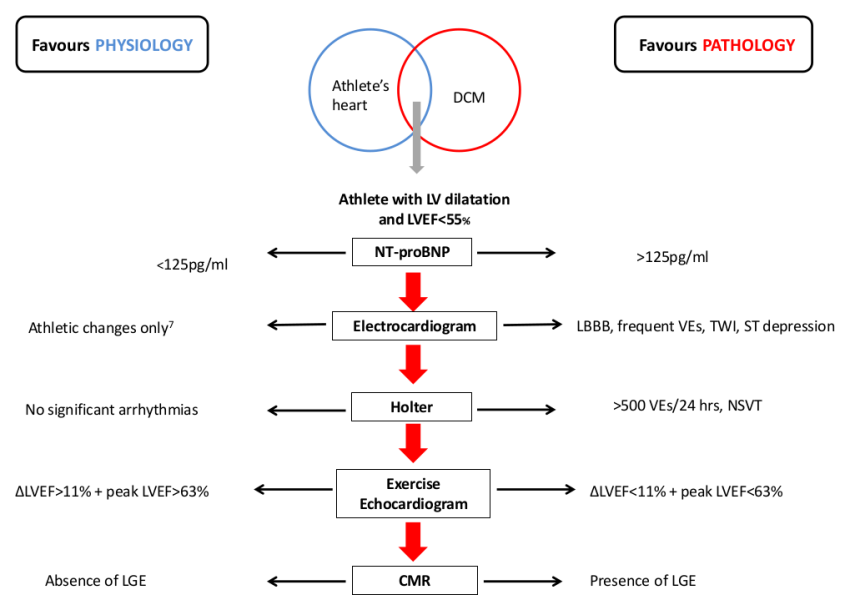

Figure 3 This figure outlines a simple algorithm to aid physicians when assessing active individuals with LV dilatation and LVEF $<55 \%$. On the left are the changes that would support physiological adaptation and on the right those that suggest pathological remodelling. The ECG was interpreted as per the international recommendations in athletes. ${ }^{7}$ CMR, cardiovascular magnetic resonance; DCM, dilated cardiomyopathy; $\mathrm{LV}$, left ventricular; LVEF, left ventricular ejection fraction; LBBB, left bundle branch block; LGE, late gadolinium enhancement; NSVT, non-sustained ventricular tachycardia; TWI, T-wave inversions; VEs, ventricular extrasystoles.

and demonstrated its utility using our data (figure 4). The two individuals without CMR have been excluded from analysis. The combination of NT-proBNP, ECG and Holter monitoring would confirm DCM in $<60 \%$ of cases. An additional exercise echocardiogram would result in a diagnosis in 31 (91.2\%) cases. A subsequent CMR could exclude pathology in another $3 \%$ of cases without impact on false positives. The algorithm has a sensitivity

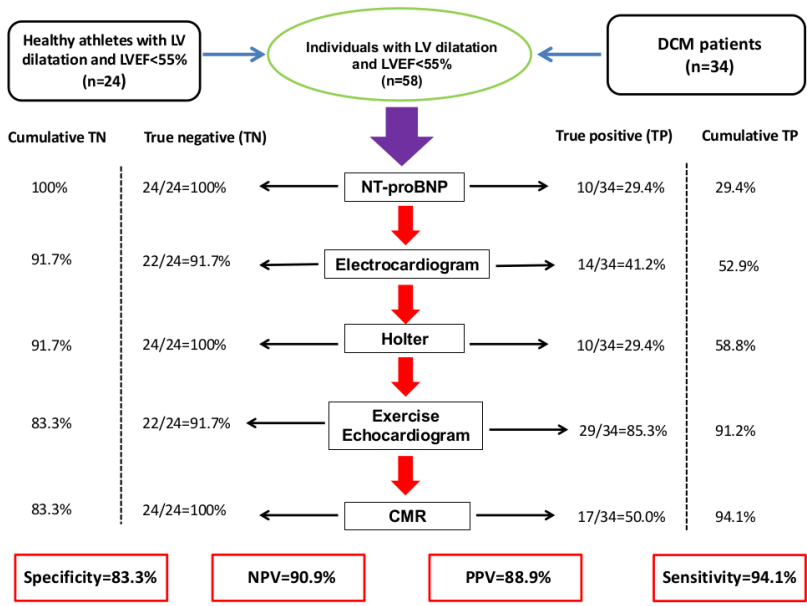

Figure 4 The figure demonstrates the utility of the stepwise clinical algorithm for differentiating between physiological adaptation and morphologically mild DCM in apparently healthy individuals with LV dilatation and LVEF $<55 \%$. The number and percentages of both cohorts with abnormal investigations is shown with the cumulative TN and TP results on the extreme right and left, respectively. The overall sensitivity of the algorithm is $94.1 \%$ with a specificity of $83.3 \%$. The PPV is $90.3 \%$ with an NPV of $94.7 \%$. CMR, cardiovascular magnetic resonance; DCM, dilated cardiomyopathy; LV, left ventricular; LVEF, left ventricular ejection fraction; NPV, negative predictive value; NT-proBNP, N-terminal pro-brain natriuretic peptide; PPV, positive predictive value; $T N$, true negative; TP, true positive. of $94.1 \%$, specificity of $83.3 \%$, positive predictive value of $88.9 \%$ and negative predictive value of $90.9 \%$. More than $70 \%$ of our patients with DCM had a normal $\mathrm{pVO}_{2}$; therefore, we would not recommend this investigation in isolation.

\section{Limitations}

Study participants were predominantly Caucasians and exclusively male; therefore, results may not readily be applicable to female athletes or the black athletic population. Given the rarity of patients with DCM who are asymptomatic and athletes in the grey zone, the numbers studied are relatively small. The algorithm was derived and assessed in the same cohort which may result in over-optimistic results; therefore, larger studies are required to validate our findings. Due to the cross-sectional nature of the study, we are unable to confidently exclude the development DCM in the grey-zone athletes in the future.

\section{Key messages}

What is already known on this subject?

- Dilated cardiomyopathy (DCM) is a recognised cause of sudden death in young athletes. It is also known that around $11 \%$ of healthy endurance athletes develop physiological left ventricular dilatation with a low/borderline left ventricular (LV) ejection fraction that may simulate DCM. Thus, a distinct grey zone exists between physiological remodelling and DCM where erroneous misinterpretation has potentially serious consequences.

\section{What might this study add?}

- We have demonstrated that failure to increase LV ejection fraction $>11 \%$ from baseline to peak exercise and inability to augment the LV ejection fraction $>63 \%$ at peak exercise during exercise stress echocardiography are suggestive of DCM with high sensitivity of around $80 \%$ and specificity $>90 \%$. Additionally, we used a composite of routine investigations to derive an algorithm to help clinicians to differentiate between athletes with a physiological increase in LV size and borderline or low baseline LV ejection fraction (grey zone) and DCM. The algorithm has a sensitivity of $94.1 \%$, specificity of $83.3 \%$, positive predictive value of $88.9 \%$ and negative predictive value of $90.9 \%$ in predicting DCM.

How might this impact on clinical practice?

- An erroneous diagnosis of DCM in an athlete with a physiologically increased LV size and borderline or low resting LV ejection fraction may lead to unnecessary disqualification from sport. Conversely, an erroneous diagnosis of athlete's heart in an individual with morphologically mild DCM deprives the individual of prognostic medications and provides false reassurance which may culminate in progressive deterioration of LV function and an exerciserelated sudden death. Our findings and proposed algorithm will aid cardiologists and sports physicians when assessing active individuals with LV dilatation and LV ejection fraction $<55 \%$ (grey zone). Although our results are promising, the numbers are relatively small and require validation in a larger cohort. 


\section{CONCLUSION}

When attempting to differentiate between physiological LV enlargement with a borderline/low baseline LVEF from mild DCM, a combination of NT-proBNP, ECG, Holter monitoring, baseline echocardiographic and CMR parameters have a modest discriminating value; however, exercise echocardiography has good sensitivity and excellent specificity.

\section{Author affiliations \\ ${ }^{1}$ Cardiology Clinical Academic Group, St. George's University Hospitals NHS Foundation Trust and Institute of Molecular and Clinical Sciences, St. George's, University of London, London, UK \\ 2Division of Cardiovascular Sciences, University of Manchester, Manchester, UK ${ }^{3}$ Research Institute of Sports and Exercise Science, Liverpool John Moores University, Liverpool, UK \\ ${ }^{4}$ Canterbury Christ Church University, Canterbury, UK \\ ${ }^{5}$ Department of Cardiology, Guy's and St Thomas' Foundation Trust, London, UK \\ ${ }^{6}$ King's College London School of Medical Education, London, UK}

Correction notice Since the online publication of this article, the authors have noticed that the affiliations were incorrect. The affiliation list has now been reduced from 9 to 6 affiliations.

Twitter Lynne Martina Millar @drlynnemillar, Harshil Dhutia @harshil_dhutia, Michael Papadakis @MichaelPapadak2 and Sanjay Sharma @SSharmacardio

Contributors All authors have been have made substantial contributions to the conception or design of the work, or the acquisition, analysis or interpretation of data and preparation of the manuscript. All authors contributed to manuscript preparation, manuscript revision, quality control and conduct of the study. LMM, ZF, $M P, M R$, JO'D, DO, MTTE, NB, ERB, GC-W, RS and SS contributed to the study design/ planning. LMM, ZF, GF, GS-F, HD, AMa, AMe, AD'S, JW, DO, RS and SS contributed to data acquisition, analysis and interpretation. LMM, GF, RS and SS contributed to statistical analysis. LMM and SS are guarantors for the study.

Funding LMM, HD, AMa and GF were funded by research grants from CRY. AD'S and AMe were funded by research grants from the British Heart Foundation.

Competing interests None declared.

Patient and public involvement Patients and/or the public were not involved in the design, conduct, reporting or dissemination plans of this research.

Patient consent for publication Not required.

Ethics approval Full ethical approval was granted by the Chelsea Research Ethics Committee, London, UK, and participants provided informed written consent.

Provenance and peer review Not commissioned; externally peer reviewed.

Data availability statement The data, analytical methods and study materials will not be made available to other researchers for the purpose of reproducing the results or replicating the procedure. Researchers interested in the data, methods or analysis can contact the corresponding author for more information.

\section{ORCID iDs}

Lynne Martina Millar http://orcid.org/0000-0002-6800-5938

Maria Teresa Tome Esteban http://orcid.org/0000-0002-8962-3524

Sanjay Sharma http://orcid.org/0000-0002-3630-6138

\section{REFERENCES}

1 Finocchiaro G, Papadakis M, Robertus J-L, et al. Etiology of sudden death in sports: insights from a United Kingdom regional registry. J Am Coll Cardiol 2016;67:2108-15

2 Abergel E, Chatellier G, Hagege AA, et al. Serial left ventricular adaptations in worldclass professional cyclists: implications for disease screening and follow-up. J Am Coll Cardiol 2004:44:144-9.
3 Galderisi M, Cardim N, D'Andrea A, et al. The multi-modality cardiac imaging approach to the athlete's heart: an expert consensus of the European association of cardiovascular imaging. Eur Heart J Cardiovasc Imaging 2015;16:353-353r.

4 La Gerche A, Baggish AL, Knuuti J, et al. Cardiac imaging and stress testing asymptomatic athletes to identify those at risk of sudden cardiac death. JACC Cardiovasc Imaging 2013;6:993-1007.

5 Lang RM, Badano LP, Mor-Avi V, et al. Recommendations for cardiac chamber quantification by echocardiography in adults: an update from the American Society of echocardiography and the European association of cardiovascular imaging. JASE 2015;28:1-39.

6 Friedmann H. Diagnostic electrocardiography and vectorcardiography. 3rd edn. New York, NY: McGraw-Hill, 1985.

7 Sharma S, Drezner JA, Baggish A, et al. International Recommendations for Electrocardiographic Interpretation in Athletes. J Am Coll Cardiol 2017;69:1057-75.

8 Zorzi A, Perazzolo Marra M, Rigato I, et al. Nonischemic left ventricular scar as a substrate of life-threatening ventricular arrhythmias and sudden cardiac death in competitive athletes. Circ Arrhythm Electrophysiol 2016;9:1-15.

9 Pedersen CT, Kay GN, Kalman J, et al. EHRA/HRS/APHRS expert consensus on ventricular arrhythmias. Heart Rhythm 2014;11:e166-96.

10 Nagueh SF, Smiseth OA, Appleton CP, et al. Recommendations for the evaluation of left ventricular diastolic function by echocardiography: an update from the American Society of echocardiography and the European association of cardiovascular imaging. Eur Heart J Cardiovasc Imaging 2016;17:1321-60.

11 Kramer CM, Barkhausen J, Flamm SD, et al. Standardized cardiovascular magnetic resonance imaging (CMR) protocols, Society for cardiovascular magnetic resonance: board of trustees Task force on standardized protocols. J Cardiovasc Magn Reson 2008;10:35.

12 Du Bois D, Du Bois EF. A formula to estimate the approximate surface area if height and weight be known. 1916. Nutrition 1989;5:303-11.

13 Nagaoka $\mathrm{H}$, Isobe $\mathrm{N}$, Kubota $\mathrm{S}$, et al. Myocardial contractile reserve as prognostic determinant in patients with idiopathic dilated cardiomyopathy without overt heart failure. Chest 1997:111:344-50.

14 Ponikowski P, Voors AA, Anker SD, et al. 2016 ESC guidelines for the diagnosis and treatment of acute and chronic heart failure. Eur Heart J 2016;37:2129-200.

15 Lippincott, Williams \& Wilkins. Principles of exercise testing and interpretation: including pathophysiology and clinical applications. 3rd edn. Philadelphia, PA: Lippincott, Williams \& Wilkins, 1999.

16 Savage DD, Seides SF, Clark CE, et al. Electrocardiographic findings in patients with obstructive and nonobstructive hypertrophic cardiomyopathy. Circulation 1978;58:402-8.

17 Papadakis M, Papadakis M, Dhutia H, et al. Electrocardiographic differentiation between 'benign T-wave inversion' and arrhythmogenic right ventricular cardiomyopathy. Europace 2019;21:332-8.

18 Beaumont A, Grace F, Richards J, et al. Left ventricular speckle Tracking-Derived cardiac strain and cardiac twist mechanics in athletes: a systematic review and metaanalysis of controlled studies. Sport Med 2016;47:1-26.

19 Otasevic P, Popovic ZB, Vasiljevic JD, et al. Head-To-Head comparison of indices of left ventricular contractile reserve assessed by high-dose dobutamine stress echocardiography in idiopathic dilated cardiomyopathy: five-year follow up. Heart 2006:92:1253-8

20 Naqvi TZ, Goel RK, Forrester JS, et al. Myocardial contractile reserve on dobutamine echocardiography predicts late spontaneous improvement in cardiac function in patients with recent onset idiopathic dilated cardiomyopathy. J Am Coll Cardiol 1999:34:1537-44.

21 Claessen G, Schnell F, Bogaert J, et al. Exercise cardiac magnetic resonance to differentiate athlete's heart from structural heart disease. Eur Heart J Cardiovasc Imaging 2018;19:1062-70.

22 Sheikh N, Papadakis M, Schnell F, et al. Clinical profile of athletes with hypertrophic cardiomyopathy. Circ Cardiovasc Imaging 2015;8:e003454.

23 Mordi I, Carrick D, Bezerra $H$, et al. $T$, and $T$, mapping for early diagnosis of dilated non-ischaemic cardiomyopathy in middle-aged patients and differentiation from normal physiological adaptation. Eur Heart J Cardiovasc Imaging 2016:17:797-803 